International Journal of Pure and Applied Mathematics

Volume 106 No. 2 2016, 495-513

ISSN: 1311-8080 (printed version); ISSN: 1314-3395 (on-line version)

url: http://www.ijpam.eu

doi: 10.12732/ijpam.v106i2.13

ijpam.eu

\title{
EXACT SOLUTIONS OF THE HIGHER-ORDER NONLINEAR SCHRÖDINGER EQUATION WITH CUBIC-QUINTIC NONLINEARITIES, SELF-STEEPING AND SELF-FREQUENCY SHIFT EFFECTS
}

\author{
M.M. Hassan ${ }^{1}$, N.H. Abdel-Wahab ${ }^{2}$, M.S. Abdel-Daym ${ }^{3} \S$ \\ 1,2,3 Mathematics Department \\ Faculty of Science \\ Minia University \\ El-Minia, EGYPT
}

\begin{abstract}
In this paper, the F-expansion method has been used to find several types of exact solutions of the higher-order nonlinear Schrödinger (HONLS) equation with cubic-quintic nonlinearities, self-steeping and self-frequency shift effects which describes the propagation of an optical pulse in optical fibers. With the aid of symbolic computation, explicit exact solutions of HONLS equation are presented which include many Jacobi elliptic function solutions, bell-shaped and kink -shaped solitary wave solutions. The properties of some solutions are shown by some figures.
\end{abstract}

AMS Subject Classification: 35Q40, 35Q55, 35Q60

Key Words: F-expansion method, Higher-order nonlinear Schrodinger equation, exact solutions

\section{Introduction}

The research area of nonlinear partial differential equations (NLPDEs) has been very active for the past few decades. The NLPDEs are widely used to describe complex phenomena in various fields of sciences, particularly in physics. Various

Received: November 19, 2015

Published: March 10, 2016

$\S_{\text {Correspondence author }}$
(C) 2016 Academic Publications, Ltd.

url: www.acadpubl.eu 
phenomena in physics can be better understood with the help of exact analytical solutions. So, the search for a mathematical algorithm to construct exact solutions of NLPDEs is an important an essential task in nonlinear sciences. In recent years, there are many effective methods that have been used to obtain exact solutions of NLPDEs such as the Bäcklund transform [1,2], the inverse scattering method [3], the tanh- function method [4], the truncated expansion method [5, 6], the Jacobi elliptic function method [7], the F-expansion method [8], the Kudryashov method [9] and other methods [10]-[13].

Propagation of picosecond pluse in optical fibers is described by the nonlinear Schrödinger (NLS) equation with cubic nonlinear term [14]. The NLS equation arises in many branches of physics and applied mathematics, such as field theory, quantum mechanics, plasma physics, condensed matter physics and nonlinear optics [14, 15]. Ebaid and Khaled [16] obtained many types of exact solutions of NLS equation. Liang et. al. [17] obtained analytical solutions to the (3+1)-dimensional NLS equation in the form of the traveling wave elliptic functions.

It is known that the propagation of picosecond or femtosecond optical pulse in fibers is described by (HONLS) equation $[18,19]$. There are many difficulties in obtaining analytical solutions for the HONLS equations. Propagation of ultrashort optical pulses in optical fibers is governed by the HONLS equation with fourth-order dispersion and cubic-quintic nonlinearity in the form [20]

$$
\begin{aligned}
i E_{z}- & \frac{\beta_{2}}{2} E_{t t}+\gamma_{1}|E|^{2} E \\
& =i \frac{\beta_{3}}{6} E_{t t t}+\frac{\beta_{4}}{24} E_{t t t t}-\gamma_{2}|E|^{4} E+i \alpha_{1}\left(|E|^{2} E\right)_{t}+i \alpha_{2} E\left(|E|^{2}\right)_{t}
\end{aligned}
$$

where $E$ is the slowly varying complex envelope of the electric field, $t$ represents the time, $z$ represents the normalized distance along the direction of propagation, $\beta_{2}$ describes group velocity dispersion, $\beta_{3}$ and $\beta_{4}$ are the third-order dispersion (TOD) and fourth-order dispersion (FOD) parameters, respectively and $\gamma_{1}$ and $\gamma_{2}$ represent the coefficients of the cubic and quintic nonlinearities of the medium. The coefficients $\alpha_{1}$ and $\alpha_{2}$ related to self-steeping (SS) and self-frequency shift arising from delayed Raman response and generally $\alpha_{2}$ should be complex. In many cases $\operatorname{Im} \alpha_{2}<<R e \alpha_{2}$, so we consider the real part of $\alpha_{2}$ as in [20]. It is important to mention that Eq. (1.1) is characterized by when $\beta_{3}=\beta_{4}=\gamma_{2}=\alpha_{1}=\alpha_{2}=0$, the standard NLS equation is given. By using an auxiliary equation, Zhang and Dai [20] have been found bright and dark optical solitons of Eq. (1.1). Moreover, the bright and dark soliton solutions are shown when $\left(\beta_{4}=\gamma_{2}=0\right)$ [21]. Recently, the exact solutions of 
HONLS equations with special parametric choices have been studied by many authors [21]-[29]. For instance, Triki and Taha [22] studied Eq. (1.1) with $\beta_{3}=\alpha_{2}=0$, Zhou et al. [23] studied Eq. (1.1) with $\alpha_{2}=0$ and Xu [27] studied Eq. (1.1) with $\beta_{3}=\alpha_{1}=\alpha_{2}=0$.

Although, exact solutions of many forms of Eq. (1.1) were reported by many authors [20, 26], we will apply the F-expansion method to obtain many types of exact solutions of HONLS equation (1.1). This paper is organized as follows: in Section 2, we give brief descriptions of the F-expansion method. In Section 3, the abundant exact solutions of Eq. (1.1) in terms of Jacobi elliptic functions ( JEFs) are obtained. Moreover, some figures are given to show the properties of some exact solutions. Finally, we summarize the main results of the paper in Section 4.

\section{Summary of the F-Expansion Method}

In this section, we would like to outline the main steps about F-expansion method [8] which summarized as follows:

Consider a NLPDE with two independent variables $z$ and $t$

$$
G\left(u, u_{t}, u_{z}, u_{t t}, u_{z z}, \ldots\right)=0 .
$$

In general, the left hand side of Eq. (2.1) is a polynomial in $u$ and its various derivatives.

Step 1: Assume that $u(z, t)=u(\xi), \xi=a(c z+t)+\xi_{0}$, where $a$ and $c$ are constants to be determined later and $\xi_{0}$ is an arbitrary constant. Then Eq. (2.1) is reduced to an ordinary differential equation (ODE)

$$
H\left(u, u^{\prime}, u^{\prime \prime}, \ldots\right)=0,
$$

where $u^{\prime}=\frac{d u}{d \xi}$ and $H$ is a polynomial of $u$ and its various derivatives.

Step 2: We seek the solutions of Eq. (2.2) in the form

$$
u(z, t)=u(\xi)=\sum_{i=0}^{N} A_{i} F^{i}(\xi),
$$

where $N$ is a positive integer that can be determined by balancing the nonlinear term (s) with the highest derivative term in Eq.(2.2) and $A_{0}, A_{i}(i=1,2, \ldots, N)$ are constants to be determined. The function $F(\xi)$ satisfies the following nonlinear ODE:

$$
\left(F^{\prime}(\xi)\right)^{2}=q_{0}+q_{2} F^{2}(\xi)+q_{4} F^{4}(\xi),
$$


where $q_{0}, q_{2}$ and $q_{4}$ are constants.

Step 3: Substituting Eq. (2.3) with Eq. (2.4) into the ODE (2.2) and setting each coefficients of the obtained polynomial to zero yields a system of algebraic equations for $A_{0}, a, A_{i}$ and $c$. Solving the system for $A_{0}, a, A_{i}$ and $c$ by using the Maple or Mathematica. Substituting the obtained coefficients into Eq. (2.3), then concentration formulas of traveling wave solutions of the NLPDE Eq. (2.1) can be obtained.

Step 4: Selecting the values of $q_{0}, q_{2}, q_{4}$ and the corresponding JEFs $F(\xi)$ and substituting them into the concentration formulas of solutions to construct more exact JEF solutions of Eq. (2.1).

The JEFs $\operatorname{sn} \xi=\operatorname{sn}(\xi, m), \operatorname{cn} \xi=\operatorname{cn}(\xi, m)$ and $\operatorname{dn} \xi=\operatorname{dn}(\xi, m)$, where $m(0<m<1)$ is the modulus of the elliptic function, are double periodic and posses the following properties:

$$
\begin{gathered}
\operatorname{sn}^{2} \xi+\mathrm{cn}^{2} \xi=1, \quad \operatorname{dn}^{2} \xi+m^{2} \operatorname{sn}^{2} \xi=1 . \\
\frac{d}{d \xi}(\operatorname{sn} \xi)=\operatorname{cn} \xi \operatorname{dn} \xi, \quad \frac{d}{d \xi}(\operatorname{cn} \xi)=-\operatorname{sn} \xi \operatorname{dn} \xi, \quad \frac{d}{d \xi}(\operatorname{dn} \xi)=-m^{2} \operatorname{sn} \xi \operatorname{cn} \xi
\end{gathered}
$$

In addition when $m \longrightarrow 1$, the functions $\operatorname{sn} \xi, \operatorname{cn} \xi$ and $\operatorname{dn} \xi$ degenerate as $\tanh \xi \operatorname{sech} \xi$ and $\operatorname{sech} \xi$, respectively, while as $m \longrightarrow 0, \operatorname{sn} \xi, \operatorname{cn} \xi$ and $\operatorname{dn} \xi$ degenerate as $\sin \xi, \cos \xi, 1$, respectively. So, we can obtain hyperbolic function solutions and trigonometric function solutions in the limit cases when $m \longrightarrow 1$ and $m \longrightarrow 0$, respectively. It is important to mention that some more properties of Jacobi elliptic functions can be found in Ref. [30].

\section{Exact JEF Solutions of Equation (1.1)}

In this section, we will use the F-expansion method to construct the exact solutions of Eq. (1.1). In order to derive some exact solutions of Eq. (1.1), we consider the solution in the form

$$
E(z, t)=u(\xi) e^{i \Theta}, \quad \xi=a(c z+t)+\xi_{0}, \quad \Theta=\Omega z+k t,
$$

where $u(\xi)$ is a real function of $\xi$ and $a, c, k$ and $\Omega$ are real constants to be determined. Substituting Eq. (3.1) into Eq. (1.1) and separating the real and the imaginary parts of the resulting complex ODE, we get

$$
\begin{aligned}
\beta_{4} a^{4} u^{\prime \prime \prime \prime}-6 a^{2}\left(2 \beta_{3} k-2 \beta_{2}+\beta_{4} k^{2}\right) u^{\prime \prime} & -\left(12 \beta_{2} k^{2}-24 \Omega-4 \beta_{3} k^{3}-\beta_{4} k^{4}\right) u \\
& -24\left(\alpha_{1} k+\gamma_{1}\right) u^{3}-24 \gamma_{2} u^{5}=0
\end{aligned}
$$




$$
a^{2}\left(\beta_{3}+\beta_{4} k\right) u^{\prime \prime \prime}-\left(6 c-6 \beta_{2} k+3 \beta_{3} k^{2}+\beta_{4} k^{3}\right) u^{\prime}+6\left(3 \alpha_{1}+2 \alpha_{2}\right) u^{2} u^{\prime}=0 .
$$

It is obviously that there are two cases to discuss.

Case 1. When $\beta_{3}+\beta_{4} k \neq 0$. Inserting the differential of Eq. (3.3) into Eq. (3.2), we have

$$
\begin{gathered}
{\left[6 a^{2}\left(\beta_{3}+\beta_{4} k\right)\left(2 \beta_{3} k-2 \beta_{2}+\beta_{4} k^{2}\right)-a^{2} \beta_{4}\left(6 c-6 \beta_{2} k+3 \beta_{3} k^{2}+\beta_{4} k^{3}\right)\right] u^{\prime \prime}} \\
+6 a^{2} \beta_{4}\left(3 \alpha_{1}+2 \alpha_{2}\right) u^{2} u^{\prime \prime}+12 a^{2} \beta_{4}\left(3 \alpha_{1}+2 \alpha_{2}\right) u\left(u^{\prime}\right)^{2}+\left(\beta_{3}+\beta_{4} k\right)\left(12 \beta_{2} k^{2}-24 \Omega\right. \\
\left.-4 \beta_{3} k^{3}-\beta_{4} k^{4}\right) u+24\left(\alpha_{1} k+\gamma_{1}\right)\left(\beta_{3}+\beta_{4} k\right) u^{3}+24 \gamma_{2}\left(\beta_{3}+\beta_{4} k\right) u^{5}=0 .
\end{gathered}
$$

By balancing the highest power nonlinear term $u^{5}$ and the highest order derivative term $u^{2} u^{\prime \prime}$ in Eq. (3.4), we obtain $N=1$. Therefore, we may choose the ansatz

$$
u(\xi)=A_{0}+A_{1} F(\xi), A_{1} \neq 0
$$

where $A_{0}, A_{1}$ are constants to be determined. Substituting Eq. (3.5) into Eq. (3.4) and using Eq. (2.4), the left hand side of Eq. (3.4) becomes a polynomial in $F(\xi)$. Setting each coefficients of $F^{j}(\xi), j=0,1,2, \ldots, 5$ to zero, we obtain a system of algebraic equations for $A_{0}, A_{1}, \Omega, a, c$ and $k$. Solving this system of algebraic equations by use of maple, we get

$$
\begin{aligned}
& A_{0}=0, \quad A_{1}= \pm a \sqrt{-\frac{\left(3 \alpha_{1}+2 \alpha_{2}\right) \beta_{4} q_{4}}{\left(k \beta_{4}+\beta_{3}\right) \gamma_{2}}}, \\
& \Omega=\frac{-1}{24 \gamma_{2}\left(k \beta_{4}+\beta_{3}\right)^{2}}\left[3 a^{4} \beta_{4}^{2}\left(3 \alpha_{1}+2 \alpha_{2}\right)^{2}\left(4 q_{0} q_{4}-3 q_{2}^{2}\right)\right. \\
& -12 a^{2} \beta_{4} q_{2}\left(3 \alpha_{1}+2 \alpha_{2}\right)\left(k \beta_{4}+\beta_{3}\right)\left(k \alpha_{1}+\gamma_{1}\right) \\
& \left.+k^{4} \beta_{4} \gamma_{2}\left(k \beta_{4}+3 \beta_{3}\right)^{2}-12 k^{2} \beta_{2} \gamma_{2}\left(k^{2} \beta_{4}^{2}+\beta_{3}^{2}\right)+4 k^{3} \beta_{3} \gamma_{2}\left(\beta_{3}^{2}-6 \beta_{2} \beta_{4}\right)\right] \text {, } \\
& c=\frac{-1}{6 \gamma_{2}\left(k \beta_{4}+\beta_{3}\right) \beta_{4}}\left[3 \beta _ { 4 } ( 3 \alpha _ { 1 } + 2 \alpha _ { 2 } ) \left(3 a^{2} \beta_{4} q_{2}\left(3 \alpha_{1}+2 \alpha_{2}\right)\right.\right. \\
& \left.+4\left(k \beta_{4}+\beta_{3}\right)\left(\gamma_{1}+k \alpha_{1}\right)\right) \\
& \left.+6 \beta_{2} \gamma_{2}\left(k \beta_{4}+\beta_{3}\right)\left(k \beta_{4}+2 \beta_{3}\right)-5 k^{3} \beta_{4}^{2} \gamma_{2}\left(k \beta_{4}+4 \beta_{3}\right)-3 k \beta_{3}^{2} \gamma_{2}\left(9 k \beta_{4}+4 \beta_{3}\right)\right] \text {, }
\end{aligned}
$$


with $a$ and $k$ are undetermined constants. Substituting these results into Eq. (3.5), we have the following formal solutions of Eq. (3.4):

$$
u(\xi)= \pm \sqrt{-\frac{\left(3 \alpha_{1}+2 \alpha_{2}\right) \beta_{4} q_{4}}{\left(k \beta_{4}+\beta_{3}\right) \gamma_{2}}} a F(\xi),
$$

with $\left(3 \alpha_{1}+2 \alpha_{2}\right)\left(k \beta_{4}+\beta_{3}\right) \beta_{4} \gamma_{2} q_{4}<0$. Substituting (3.9) into the ODEs (3.2) and (3.3) and setting each coefficients of the obtained polynomials to zero yields a set of algebraic equations for $a$ and $k$. Solving these equations, we obtain

$$
\begin{gathered}
a= \pm \frac{1}{\beta_{4}} \sqrt{\frac{3\left[\beta_{4}\left(3 \alpha_{1}^{2}+8 \alpha_{1} \alpha_{2}+4 \alpha_{2}^{2}\right)-\gamma_{2}\left(\beta_{3}^{2}+2 \beta_{2} \beta_{4}\right)-2 \lambda\right]}{5 \gamma_{2} q_{2}}}, \quad k=\frac{-\beta_{3} \gamma_{2}-\left(3 \alpha_{1}+2 \alpha_{2}\right) \sqrt{\beta_{4} \gamma_{2}}}{\gamma_{2} \beta_{4}}, \\
a= \pm \frac{1}{\beta_{4}} \sqrt{\frac{3\left[\beta_{4}\left(3 \alpha_{1}^{2}+8 \alpha_{1} \alpha_{2}+4 \alpha_{2}^{2}\right)-\gamma_{2}\left(\beta_{3}^{2}+2 \beta_{2} \beta_{4}\right)+2 \lambda\right]}{5 \gamma_{2} q_{2}}}, \quad k=\frac{-\beta_{3} \gamma_{2}+\left(3 \alpha_{1}+2 \alpha_{2}\right) \sqrt{\beta_{4} \gamma_{2}}}{\gamma_{2} \beta_{4}} \\
\gamma_{2} \beta_{4}>0, \quad 3 \alpha_{1}+2 \alpha_{2}
\end{gathered}
$$

with

$$
\lambda=\left(\alpha_{1} \beta_{3}-\beta_{4} \gamma_{1}\right) \sqrt{\gamma_{2} \beta_{4}}, \quad \alpha_{1} \beta_{3}-\beta_{4} \gamma_{1} \neq 0 .
$$

Therefore, we can obtain abundant JEF solutions of Eq. (1.1) by selecting the values of $q_{0}, q_{2}, q_{4}$ and the corresponding JEFs $F(\xi)$ as follows:

Case 1.1: When $q_{0}=1, q_{2}=-1-m^{2}, q_{4}=m^{2}$, Eq. (2.4) has the solution $F(\xi)=\operatorname{sn} \xi$. So, we obtain the following exact JEF solutions of Eq. (1.1):

$E_{1,2}(z, t)= \pm m a \sqrt{-\frac{\left(3 \alpha_{1}+2 \alpha_{2}\right) \beta_{4}}{\left(k \beta_{4}+\beta_{3}\right) \gamma_{2}}} e^{i \Theta} \operatorname{sn} \xi, \quad \xi=a(c z+t)+\xi_{0}, \quad \Theta=\Omega z+k t$,

where $a$ and $k$ are given in Eq. (3.10) with the corresponding value of $q_{2}$. The intensity of these solutions is given by

$$
\left|E_{1,2}(z, t)\right|^{2}=\frac{-m^{2} a^{2}\left(3 \alpha_{1}+2 \alpha_{2}\right) \beta_{4}}{\left(k \beta_{4}+\beta_{3}\right) \gamma_{2}} \operatorname{sn}^{2} \xi
$$

Case 1.2: When $q_{0}=1-m^{2}, q_{2}=2 m^{2}-1, q_{4}=-m^{2}, F(\xi)=\mathrm{cn} \xi$, we obtain the exact solutions of Eq. (1.1)

$$
E_{3,4}(z, t)= \pm \sqrt{\frac{\left(3 \alpha_{1}+2 \alpha_{2}\right) \beta_{4}}{\left(k \beta_{4}+\beta_{3}\right) \gamma_{2}}} m a e^{i \Theta} \mathrm{cn} \xi
$$

Case 1.3: When $q_{0}=m^{2}-1, q_{2}=2-m^{2}, q_{4}=-1, F(\xi)=\operatorname{dn} \xi$, we obtain the following exact solutions of Eq. (1.1):

$$
E_{5,6}(z, t)= \pm \sqrt{\frac{\left(3 \alpha_{1}+2 \alpha_{2}\right) \beta_{4}}{\left(k \beta_{4}+\beta_{3}\right) \gamma_{2}}} a e^{i \Theta} \operatorname{dn} \xi
$$


Case 1.4: When $q_{0}=\frac{1}{4}, q_{2}=\frac{m^{2}-2}{2}, q_{4}=\frac{m^{4}}{4}$, we have $F(\xi)=\frac{\operatorname{sn} \xi}{1 \pm \operatorname{dn} \xi}$. Therefore, we obtain the following exact solutions of Eq. (1.1):

$$
E_{7,8}(z, t)= \pm \sqrt{-\frac{\left(3 \alpha_{1}+2 \alpha_{2}\right) \beta_{4}}{\left(k \beta_{4}+\beta_{3}\right) \gamma_{2}}} \frac{m^{2} a e^{i \Theta} \operatorname{sn} \xi}{2(1 \pm \operatorname{dn} \xi)}
$$

Case 1.5: When $q_{0}=\frac{m^{2}-1}{4}, q_{2}=\frac{m^{2}+1}{2}, q_{4}=\frac{m^{2}-1}{4}$ we have $F(\xi)=\frac{\mathrm{dn} \xi}{1 \pm m \operatorname{sn} \xi}$. Therefore, we construct the following solutions of Eq. (1.1):

$$
E_{9,10}(z, t)= \pm \sqrt{-\frac{\left(3 \alpha_{1}+2 \alpha_{2}\right)\left(m^{2}-1\right) \beta_{4}}{\left(k \beta_{4}+\beta_{3}\right)\left(m^{2}-1\right) \gamma_{2}}} \frac{a e^{i \Theta} \mathrm{dn} \xi}{2(1 \pm m \operatorname{sn} \xi)}
$$

Case 1.6: When $q_{0}=\frac{1-m^{2}}{4}, q_{2}=\frac{m^{2}+1}{2}, q_{4}=\frac{1-m^{2}}{4}, F(\xi)=\frac{\mathrm{cn} \xi}{1 \pm \operatorname{sn} \xi}$, we obtain

$$
E_{11,12}(z, t)= \pm \sqrt{-\frac{\left(3 \alpha_{1}+2 \alpha_{2}\right)\left(1-m^{2}\right) \beta_{4}}{\left(k \beta_{4}+\beta_{3}\right)\left(1-m^{2}\right) \gamma_{2}}} \frac{a e^{i \Theta} \operatorname{cn} \xi}{2(1 \pm \operatorname{sn} \xi)}
$$

Case 1.7: When $q_{0}=\frac{-\left(1-m^{2}\right)^{2}}{4}, q_{2}=\frac{m^{2}+1}{2}, q_{4}=\frac{-1}{4}, F(\xi)=(m \operatorname{cn} \xi \pm \operatorname{dn} \xi)$, we obtain

$$
E_{13,14}(z, t)= \pm \sqrt{\frac{\left(3 \alpha_{1}+2 \alpha_{2}\right) \beta_{4}}{\left(k \beta_{4}+\beta_{3}\right) \gamma_{2}}} e^{i \Theta} \frac{a}{2}(m \operatorname{cn} \xi \pm \operatorname{dn} \xi)
$$

Case 1.8: When $q_{0}=\frac{1}{4}, q_{2}=\frac{m^{2}+1}{2}, q_{4}=\frac{\left(1-m^{2}\right)^{2}}{4}$, we have $F(\xi)=\frac{\operatorname{sn} \xi}{\operatorname{cn} \xi \pm \operatorname{dn} \xi}$. Thus, we construct the following solutions of Eq. (1.1):

$$
E_{15,16}(z, t)= \pm \sqrt{-\frac{\left(3 \alpha_{1}+2 \alpha_{2}\right) \beta_{4}}{\left(k \beta_{4}+\beta_{3}\right) \gamma_{2}}} \frac{\left(1-m^{2}\right) a e^{i \Theta} \operatorname{sn} \xi}{2(\operatorname{cn} \xi \pm \operatorname{dn} \xi)} .
$$

Case 1.9: When $q_{0}=1, q_{2}=2-m^{2}, q_{4}=1-m^{2}, F(\xi)=\operatorname{sc} \xi$, we obtain the following exact solutions of Eq. (1.1):

$$
E_{17,18}(z, t)= \pm \sqrt{-\frac{\left(3 \alpha_{1}+2 \alpha_{2}\right)\left(1-m^{2}\right) \beta_{4}}{\left(k \beta_{4}+\beta_{3}\right)\left(1-m^{2}\right) \gamma_{2}}} a e^{i \Theta} \operatorname{sc} \xi .
$$

Case 1.10: When $q_{0}=m^{2}, q_{2}=-m^{2}-1, q_{4}=1, F(\xi)=$ ns $\xi$ or $F(\xi)=\operatorname{dc} \xi$, we obtain the following exact solutions of Eq. (1.1):

$$
E_{19,20}(z, t)= \pm \sqrt{-\frac{\left(3 \alpha_{1}+2 \alpha_{2}\right) \beta_{4}}{\left(k \beta_{4}+\beta_{3}\right) \gamma_{2}}} a e^{i \Theta} \mathrm{ns} \xi
$$




$$
E_{21,22}(z, t)= \pm \sqrt{-\frac{\left(3 \alpha_{1}+2 \alpha_{2}\right) \beta_{4}}{\left(k \beta_{4}+\beta_{3}\right) \gamma_{2}}} a e^{i \Theta} \mathrm{dc} \xi .
$$

Case 1.11: When $q_{0}=m^{4}-2 m^{3}+m^{2}, q_{2}=6 m-m^{2}-1, q_{4}=\frac{-4}{m}$, then the solution of Eq. (2.4) is $F(\xi)=\frac{m \operatorname{cn} \xi \operatorname{dn} \xi}{\left(m \mathrm{sn}^{2} \xi+1\right)}$, and the solutions of Eq. (1.1) are

$$
E_{23,24}(z, t)= \pm \sqrt{\frac{m\left(3 \alpha_{1}+2 \alpha_{2}\right) \beta_{4}}{\left(k \beta_{4}+\beta_{3}\right) \gamma_{2}}} \frac{2 a e^{i \Theta} \operatorname{cn} \xi \operatorname{dn} \xi}{\left(1+m \mathrm{sn}^{2} \xi\right)}
$$

where $\Omega, c$ are given in Eq. (3.7) and Eq. (3.8), respectively and $a, k$ are given in Eq. (3.10) with the corresponding values of $q_{0}, q_{2}, q_{4}$.

When the modulus $m \rightarrow 1$, the JEFs solutions in equations (3.12)-(3.23) degenerate to the following hyperbolic function solutions:

$$
\begin{gathered}
E_{25,26}(z, t)= \pm \sqrt{-\frac{\left(3 \alpha_{1}+2 \alpha_{2}\right) \beta_{4}}{\left(k \beta_{4}+\beta_{3}\right) \gamma_{2}}} a e^{i \Theta} \tanh \xi \\
E_{27,28}(z, t)= \pm \sqrt{\frac{\left(3 \alpha_{1}+2 \alpha_{2}\right) \beta_{4}}{\left(k \beta_{4}+\beta_{3}\right) \gamma_{2}}} a e^{i \Theta} \operatorname{sech} \xi \\
E_{29,30}(z, t)= \pm \sqrt{-\frac{\left(3 \alpha_{1}+2 \alpha_{2}\right) \beta_{4}}{\left(k \beta_{4}+\beta_{3}\right) \gamma_{2}}} \frac{a e^{i \Theta} \tanh \xi}{2(1 \pm \operatorname{sech} \xi)} \\
E_{31,32}(z, t)= \pm \sqrt{\frac{\left(3 \alpha_{1}+2 \alpha_{2}\right) \beta_{4}}{\left(k \beta_{4}+\beta_{3}\right) \gamma_{2}}} e^{i \Theta} \frac{2 a \operatorname{sech}^{2}(\xi)}{\left[\tanh ^{2}(\xi)+1\right]}
\end{gathered}
$$

the solutions in Eq. (3.24) and Eq. (3.25) are called dark and bright soliton solutions, respectively. One can note that for the bright solitary wave solutions to exist, it is necessary from the expression $A_{1}$ in Eq. (3.6) that $\left(3 \alpha_{1}+2 \alpha_{2}\right)\left(k \beta_{4}+\beta_{3}\right) \beta_{4} \gamma_{2}>0$ and the corresponding intensity of the bright solitary wave solutions takes the form:

$$
\left|E_{27,28}(z, t)\right|^{2}=\frac{\left(3 \alpha_{1}+2 \alpha_{2}\right) \beta_{4}}{\left(k \beta_{4}+\beta_{3}\right) \gamma_{2}} a^{2} \operatorname{sech}^{2} \xi
$$

Also, for the dark solitary wave solutions the necessary condition to exist is $\left(3 \alpha_{1}+2 \alpha_{2}\right)\left(k \beta_{4}+\beta_{3}\right) \beta_{4} \gamma_{2}<0$ and the corresponding intensity is given by

$$
\left|E_{25,26}(z, t)\right|^{2}=\frac{-\left(3 \alpha_{1}+2 \alpha_{2}\right) \beta_{4}}{\left(k \beta_{4}+\beta_{3}\right) \gamma_{2}} a^{2} \tanh ^{2} \xi .
$$


Clearly one can conclude that the existence of bright and the dark solitary wave solutions depends on the coefficients of the right terms in Eq. (1.1).

Based on the above calculations, the periodic properties of the intensity of solutions (3.12), (3.13) and (3.15) are shown in Fig.1 in terms of JEFs with different parameters. Also, we shall discuss the intensity of bright and dark solitary wave solutions given in (3.25) and (3.24) for different parameters in Fig. 2 and Fig. 3, respectively. Fig. 2(a), shows the intensity profile of the bright solitary wave solutions with the choice of parameters as $\beta_{2}=2, \beta_{3}=$ $0.9, \beta_{4}=0.6, \gamma_{1}=\gamma_{2}=1.5, \alpha_{1}=\alpha_{2}=0.3$ which satisfy the constraint relation. In Fig. 2(b), we have plotted the intensity of the bright solitary wave solutions at $t=1$ with different values of $\gamma_{2}$. It is observed that with the increase of $\gamma_{2}$ the amplitude of the intensity decreases while the width increases. In Fig. $2(\mathrm{c})$, we have studied the effect of the parameter $\beta_{4}$ on the intensity profile of the bright solitary wave solutions (3.25) at $t=1$. It is observe that with the increase of $\beta_{4}$ the amplitude decreases and the width increases. In Fig. 3(a), we have plotted the intensity profile of the dark solitary wave solution with the same parameters as in Fig. 2(a) but $\alpha_{1}=\alpha_{2}=-0.3$. In Fig. 3(b), we have plotted the intensity of the dark solitary wave solutions at $t=1$ with different values of $\gamma_{2}$. It is seen as $\gamma_{2}$ increases the amplitude of the intensity decreases while the width increases. In Fig. 3(c), we have investigated the effect of the parameter $\beta_{4}$ on the intensity profile of the dark solitary wave solutions. It is shown that the increase of $\beta_{4}$ leads to increasing the amplitude decreases while the width increases.

When $m \rightarrow 0$, the solutions given in equations (3.12)-(3.23) reduced to the triangular function solutions as follows:

$$
\begin{aligned}
& E_{33,34}(z, t)= \pm \sqrt{-\frac{\left(3 \alpha_{1}+2 \alpha_{2}\right) \beta_{4}}{\left(k \beta_{4}+\beta_{3}\right) \gamma_{2}}} a e^{i \Theta} \sec \xi \\
& E_{35,36}(z, t)= \pm \sqrt{-\frac{\left(3 \alpha_{1}+2 \alpha_{2}\right) \beta_{4}}{\left(k \beta_{4}+\beta_{3}\right) \gamma_{2}}} a e^{i \Theta} \tan \xi .
\end{aligned}
$$

Case 2: When $\beta_{3}+\beta_{4} k=0$, then we have

$$
k=-\frac{\beta_{3}}{\beta_{4}},
$$

Eq. (3.3) becomes

$$
\left[\left(6 c-6 \beta_{2} k+3 \beta_{3} k^{2}+\beta_{4} k^{3}\right)-6\left(3 \alpha_{1}+2 \alpha_{2}\right) u^{2}\right] u^{\prime}=0 .
$$


So, we obtain

$$
6 c-6 \beta_{2} k+3 \beta_{3} k^{2}+\beta_{4} k^{3}=0, \quad 3 \alpha_{1}+2 \alpha_{2}=0,
$$

from Eq. (3.32) and Eq. (3.33), we get

$$
c=\frac{-\left(3 \beta_{2} \beta_{3} \beta_{4}+\beta_{3}^{3}\right)}{3 \beta_{4}^{2}} .
$$

Substituting Eq. (3.32) into Eq. (3.2) yields

$$
\begin{aligned}
\beta_{4}^{4} a^{4} u^{\prime \prime \prime \prime}+6 a^{2} \beta_{4}^{2}\left(2 \beta_{2} \beta_{4}+\beta_{3}^{2}\right) u^{\prime \prime}-3\left(4 \beta_{2} \beta_{3}^{2} \beta_{4}-8 \Omega \beta_{4}^{3}+\beta_{3}^{4}\right) u \\
-24 \beta_{4}^{2}\left(\gamma_{1} \beta_{4}-\alpha_{1} \beta_{3}\right) u^{3}-24 \beta_{4}^{3} \gamma_{2} u^{5}=0 .
\end{aligned}
$$

By balancing the nonlinear term and the highest order derivative term in Eq. (3.35), we find that $N=1$. So, we may choose the exact solutions of Eq. (3.35) in the form (3.5). Substituting this solution with Eq. (2.4) into Eq. (3.35) and setting the coefficients of the obtained polynomial equal to zero, we get a system of algebraic equations for $A_{0}, A_{1}, a$, and $\Omega$. Solving this system of algebraic equations by maple, yields the following solutions:

$$
\begin{aligned}
& a= \pm \frac{1}{5} \frac{\sqrt{15} \sqrt{\gamma_{2} q_{2}\left(-2 \beta_{2} \beta_{4} \gamma_{2}-\beta_{3}^{2} \gamma_{2}+2 \lambda\right)}}{\gamma_{2} q_{2} \beta_{4}}, \\
& A_{0}=0, \quad A_{1}= \pm \frac{1}{\beta_{4}} \sqrt{\frac{-q_{4}\left[6 \beta_{4}\left(\alpha_{1} \beta_{3}-\beta_{4} \gamma_{1}\right)^{2}-\left(6 \beta_{2} \beta_{4}+3 \beta_{3}^{2}\right) \lambda\right]}{5 \gamma_{2} q_{2}\left(\alpha_{1} \beta_{3}-\beta_{4} \gamma_{1}\right)}}, \\
& \Omega=\frac{1}{50\left(\gamma_{2} q_{2}{ }^{2} \beta_{4}{ }^{3}\right)}\left(( 3 6 \beta _ { 2 } \beta _ { 4 } q _ { 0 } q _ { 4 } - 1 2 \beta _ { 2 } \beta _ { 4 } q _ { 2 } { } ^ { 2 } + 1 8 \beta _ { 3 } { } ^ { 2 } q _ { 0 } q _ { 4 } - 6 \beta _ { 3 } { } ^ { 2 } q _ { 2 } { } ^ { 2 } ) \left(-2 \beta_{2} \beta_{4} \gamma_{2}\right.\right. \\
& \left.-\beta_{3}{ }^{2} \gamma_{2}+2 \lambda\right)-36 \alpha_{1}^{2} \beta_{3}^{2} \beta_{4} q_{0} q_{4}-3 \alpha_{1}^{2} \beta_{3}^{2} \beta_{4} q_{2}{ }^{2}+72 \alpha_{1} \beta_{3} \beta_{4}{ }^{2} \gamma_{1} q_{0} q_{4} \\
& +6 \alpha_{1} \beta_{3} \beta_{4}{ }^{2} \gamma_{1} q_{2}^{2}+36 \beta_{2}^{2} \beta_{4}{ }^{2} \gamma_{2} q_{0} q_{4}+3 \beta_{2}{ }^{2} \beta_{4}{ }^{2} \gamma_{2} q_{2}{ }^{2}+36 \beta_{2} \beta_{3}{ }^{2} \beta_{4} \gamma_{2} q_{0} q_{4} \\
& \left.+28 \beta_{2} \beta_{3}{ }^{2} \beta_{4} \gamma_{2} q_{2}{ }^{2}+9 \beta_{3}{ }^{4} \gamma_{2} q_{0} q_{4}+7 \beta_{3}{ }^{4} \gamma_{2} q_{2}{ }^{2}-36 \beta_{4}{ }^{3} \gamma_{1}{ }^{2} q_{0} q_{4}-3 \beta_{4}{ }^{3} \gamma_{1}{ }^{2} q_{2}{ }^{2}\right) \text {, } \\
& a= \pm \frac{1}{5} \frac{\sqrt{-15 \gamma_{2} q_{2}\left(2 \beta_{2} \beta_{4} \gamma_{2}+\beta_{3}{ }^{2} \gamma_{2}+2 \lambda\right)}}{\gamma_{2} q_{2} \beta_{4}} \\
& A_{0}=0, \quad A_{1}= \pm \frac{1}{\beta_{4}} \sqrt{\frac{-q_{4}\left[6 \beta_{4}\left(\alpha_{1} \beta_{3}-\beta_{4} \gamma_{1}\right)^{2}+\left(6 \beta_{2} \beta_{4}+3 \beta_{3}{ }^{2}\right) \lambda\right]}{5 \gamma_{2} q_{2}\left(\alpha_{1} \beta_{3}-\beta_{4} \gamma_{1}\right)}}, \\
& \Omega=\frac{1}{50\left(\gamma_{2} q_{2}{ }^{2} \beta_{4}{ }^{3}\right)}\left(( - 3 6 \beta _ { 2 } \beta _ { 4 } q _ { 0 } q _ { 4 } + 1 2 \beta _ { 2 } \beta _ { 4 } q _ { 2 } { } ^ { 2 } - 1 8 \beta _ { 3 } { } ^ { 2 } q _ { 0 } q _ { 4 } + 6 \beta _ { 3 } { } ^ { 2 } q _ { 2 } { } ^ { 2 } ) \left(2 \beta_{2} \beta_{4} \gamma_{2}\right.\right. \\
& \left.+\beta_{3}{ }^{2} \gamma_{2}+2 \lambda\right)-36 \alpha_{1}{ }^{2} \beta_{3}{ }^{2} \beta_{4} q_{0} q_{4}-3 \alpha_{1}{ }^{2} \beta_{3}{ }^{2} \beta_{4} q_{2}{ }^{2}+72 \alpha_{1} \beta_{3} \beta_{4}{ }^{2} \gamma_{1} q_{0} q_{4} \\
& +6 \alpha_{1} \beta_{3} \beta_{4}{ }^{2} \gamma_{1} q_{2}{ }^{2}+36 \beta_{2}{ }^{2} \beta_{4}{ }^{2} \gamma_{2} q_{0} q_{4}+3 \beta_{2}{ }^{2} \beta_{4}{ }^{2} \gamma_{2} q_{2}{ }^{2}+36 \beta_{2} \beta_{3}{ }^{2} \beta_{4} \gamma_{2} q_{0} q_{4} \\
& \left.+28 \beta_{2} \beta_{3}{ }^{2} \beta_{4} \gamma_{2} q_{2}{ }^{2}+9 \beta_{3}{ }^{4} \gamma_{2} q_{0} q_{4}+7 \beta_{3}{ }^{4} \gamma_{2} q_{2}{ }^{2}-36 \beta_{4}{ }^{3} \gamma_{1}{ }^{2} q_{0} q_{4}-3 \beta_{4}{ }^{3} \gamma_{1}{ }^{2} q_{2}{ }^{2}\right) \text {, }
\end{aligned}
$$


Thus, the solutions of Eq. (3.35) are given by

$$
u(\xi)= \pm \frac{1}{\beta_{4}} \sqrt{\frac{-q_{4}\left[6 \beta_{4}\left(\alpha_{1} \beta_{3}-\beta_{4} \gamma_{1}\right)^{2}-\left(6 \beta_{2} \beta_{4}+3 \beta_{3}^{2}\right) \lambda\right]}{5 \gamma_{2} q_{2}\left(\alpha_{1} \beta_{3}-\beta_{4} \gamma_{1}\right)}} F(\xi)
$$

with $\gamma_{2} \beta_{4}>0$, and $q_{4} q_{2} \gamma_{2}\left[2 \beta_{4}\left(\alpha_{1} \beta_{3}-\beta_{4} \gamma_{1}\right)-\left(2 \beta_{2} \beta_{4}+\beta_{3}^{2}\right) \sqrt{\gamma_{2} \beta_{4}}\right]<0$ and $k, c, a, \Omega$ and $\lambda$ are given by Eq. (3.32), Eq. (3.34), (3.36) and (3.11), respectively and

$$
u(\xi)= \pm \frac{1}{\beta_{4}} \sqrt{\frac{-q_{4}\left[6 \beta_{4}\left(\alpha_{1} \beta_{3}-\beta_{4} \gamma_{1}\right)^{2}+\left(6 \beta_{2} \beta_{4}+3 \beta_{3}^{2}\right) \lambda\right]}{5 \gamma_{2} q_{2}\left(\alpha_{1} \beta_{3}-\beta_{4} \gamma_{1}\right)}} F(\xi),
$$

where $\gamma_{2} \beta_{4}>0$, and $q_{4} q_{2} \gamma_{2}\left[2 \beta_{4}\left(\alpha_{1}, \beta_{3}-\beta_{4} \gamma_{1}\right)+\left(2 \beta_{2} \beta_{4}+\beta_{3}^{2}\right) \sqrt{\gamma_{2} \beta_{4}}\right]<0$ and $k, c, a, \Omega$ and $\lambda$ are given by Eq. (3.32), Eq. (3.34), (3.37) and (3.11), respectively.

In this case, we obtain the exact solutions of Eq. (1.1) in terms of JEFs by selecting the values of $q_{0}, q_{2}, q_{4}$ and the corresponding JEFs $F(\xi)$ as follows:

Case 2.1: If $q_{0}=1, q_{2}=-1-m^{2}, q_{4}=m^{2}$, then Eq. (2.4) has the solution $F(\xi)=\operatorname{sn} \xi$ and the solutions of Eq. (1.1) are

$$
\begin{aligned}
& E_{37,38}(z, t)= \pm \frac{m}{\beta_{4}} \sqrt{\frac{\left[6 \beta_{4}\left(\alpha_{1} \beta_{3}-\beta_{4} \gamma_{1}\right)^{2}-\left(6 \beta_{2} \beta_{4}+3 \beta_{3}^{2}\right) \lambda\right]}{5 \gamma_{2}\left(1+m^{2}\right)\left(\alpha_{1} \beta_{3}-\beta_{4} \gamma_{1}\right)}} e^{i \Theta} \operatorname{sn} \xi \\
& E_{39,40}(z, t)= \pm \frac{m}{\beta_{4}} \sqrt{\frac{\left[6 \beta_{4}\left(\alpha_{1} \beta_{3}-\beta_{4} \gamma_{1}\right)^{2}+\left(6 \beta_{2} \beta_{4}+3 \beta_{3}^{2}\right) \lambda\right]}{5 \gamma_{2}\left(1+m^{2}\right)\left(\alpha_{1} \beta_{3}-\beta_{4} \gamma_{1}\right)}} e^{i \Theta} \operatorname{sn} \xi
\end{aligned}
$$

where $\Omega$ and $a$ in Eq. (3.40) and Eq. (3.41) are given in Eq. (3.36) and Eq. (3.37), respectively with the corresponding values of $q_{0}, q_{2}, q_{4}$ and $k, c$ are given by Eq. (3.32) and Eq. (3.34).

Case 2.2: When $q_{0}=1-m^{2}, q_{2}=2 m^{2}-1, q_{4}=-m^{2}$, then Eq. (2.4) has the solution $F(\xi)=\operatorname{cn} \xi$ from which we get

$$
\begin{aligned}
& E_{41,42}(z, t)= \pm \frac{m}{\beta_{4}} \sqrt{\frac{\left[6 \beta_{4}\left(\alpha_{1} \beta_{3}-\beta_{4} \gamma_{1}\right)^{2}-\left(6 \beta_{2} \beta_{4}+3 \beta_{3}^{2}\right) \lambda\right]}{5 \gamma_{2}\left(2 m^{2}-1\right)\left(\alpha_{1} \beta_{3}-\beta_{4} \gamma_{1}\right)}} e^{i \Theta} \mathrm{cn} \xi \\
& E_{43,44}(z, t)= \pm \frac{m}{\beta_{4}} \sqrt{\frac{\left[6 \beta_{4}\left(\alpha_{1} \beta_{3}-\beta_{4} \gamma_{1}\right)^{2}+\left(6 \beta_{2} \beta_{4}+3 \beta_{3}^{2}\right) \lambda\right]}{5 \gamma_{2}\left(2 m^{2}-1\right)\left(\alpha_{1} \beta_{3}-\beta_{4} \gamma_{1}\right)}} e^{i \Theta} \mathrm{cn} \xi
\end{aligned}
$$


Case 2.3: When $q_{0}=m^{2}-1, q_{2}=2-m^{2}, q_{4}=-1, F(\xi)=\operatorname{dn} \xi$, we obtain

$$
\begin{aligned}
& E_{45,46}(z, t)= \pm \frac{1}{\beta_{4}} \sqrt{\frac{\left[6 \beta_{4}\left(\alpha_{1} \beta_{3}-\beta_{4} \gamma_{1}\right)^{2}-\left(6 \beta_{2} \beta_{4}+3 \beta_{3}^{2}\right) \lambda\right]}{5 \gamma_{2}\left(2-m^{2}\right)\left(\alpha_{1} \beta_{3}-\beta_{4} \gamma_{1}\right)}} e^{i \Theta} \operatorname{dn} \xi \\
& E_{47,48}(z, t)= \pm \frac{1}{\beta_{4}} \sqrt{\frac{\left[6 \beta_{4}\left(\alpha_{1} \beta_{3}-\beta_{4} \gamma_{1}\right)^{2}+\left(6 \beta_{2} \beta_{4}+3 \beta_{3}^{2}\right) \lambda\right]}{5 \gamma_{2}\left(2-m^{2}\right)\left(\alpha_{1} \beta_{3}-\beta_{4} \gamma_{1}\right)}} e^{i \Theta} \operatorname{dn} \xi
\end{aligned}
$$

Case 2.4: When $q_{0}=1, q_{2}=2-m^{2}, q_{4}=1-m^{2}, F(\xi)=$ sc $\xi$, we obtain

$$
\begin{aligned}
& E_{49,50}(z, t)= \pm \frac{1}{\beta_{4}} \sqrt{\frac{\left(m^{2}-1\right)\left[6 \beta_{4}\left(\alpha_{1} \beta_{3}-\beta_{4} \gamma_{1}\right)^{2}-\left(6 \beta_{2} \beta_{4}+3 \beta_{3}^{2}\right) \lambda\right]}{5 \gamma_{2}\left(2-m^{2}\right)\left(\alpha_{1} \beta_{3}-\beta_{4} \gamma_{1}\right)}} e^{i \Theta} \mathrm{sc} \xi \\
& E_{51,52}(z, t)= \pm \frac{1}{\beta_{4}} \sqrt{\frac{\left(m^{2}-1\right)\left[6 \beta_{4}\left(\alpha_{1} \beta_{3}-\beta_{4} \gamma_{1}\right)^{2}+\left(6 \beta_{2} \beta_{4}+3 \beta_{3}^{2}\right) \lambda\right]}{5 \gamma_{2}\left(2-m^{2}\right)\left(\alpha_{1} \beta_{3}-\beta_{4} \gamma_{1}\right)}} e^{i \Theta} \operatorname{sc} \xi
\end{aligned}
$$

Case 2.5: If $q_{0}=\frac{1}{4}, q_{2}=\frac{m^{2}-2}{2}, q_{4}=\frac{m^{4}}{4}, F(\xi)=\frac{\mathrm{sn} \xi}{1 \pm \mathrm{dn} \xi}$, thus yields the exact solutions of Eq. (1.1) as follows:

$$
\begin{aligned}
& E_{53,54}(z, t)= \pm \frac{m^{2}}{2 \beta_{4}} \sqrt{\frac{2\left[6 \beta_{4}\left(\alpha_{1} \beta_{3}-\beta_{4} \gamma_{1}\right)^{2}-\left(6 \beta_{2} \beta_{4}+3 \beta_{3}^{2}\right) \lambda\right]}{5 \gamma_{2}\left(2-m^{2}\right)\left(\alpha_{1} \beta_{3}-\beta_{4} \gamma_{1}\right)}} \frac{e^{i \Theta} \operatorname{sn} \xi}{(1 \pm \operatorname{dn} \xi)} \\
& E_{55,56}(z, t)= \pm \frac{m^{2}}{2 \beta_{4}} \sqrt{\frac{2\left[6 \beta_{4}\left(\alpha_{1} \beta_{3}-\beta_{4} \gamma_{1}\right)^{2}+\left(6 \beta_{2} \beta_{4}+3 \beta_{3}^{2}\right) \lambda\right]}{5 \gamma_{2}\left(2-m^{2}\right)\left(\alpha_{1} \beta_{3}-\beta_{4} \gamma_{1}\right)}} \frac{e^{i \Theta} \operatorname{sn} \xi}{(1 \pm \operatorname{dn} \xi)}
\end{aligned}
$$

case 2.6: If $q_{0}=\frac{m^{2}-1}{4}, q_{2}=\frac{m^{2}+1}{2}, q_{4}=\frac{m^{2}-1}{4}, F(\xi)=\frac{\operatorname{dn} \xi}{1 \pm m \operatorname{sn} \xi}$, thus yields the exact solutions of Eq. (1.1) as follows:

$$
\begin{aligned}
& E_{57,58}= \pm \frac{1}{\beta_{4}} \sqrt{\frac{\left(1-m^{2}\right)\left[6 \beta_{4}\left(\alpha_{1} \beta_{3}-\beta_{4} \gamma_{1}\right)^{2}-\left(6 \beta_{2} \beta_{4}+3 \beta_{3}^{2}\right) \lambda\right]}{10 \gamma_{2}\left(1+m^{2}\right)\left(\alpha_{1} \beta_{3}-\beta_{4} \gamma_{1}\right)}} \frac{e^{i \Theta} \operatorname{dn} \xi}{(1 \pm m \operatorname{sn} \xi)}, \\
& E_{59,60}= \pm \frac{1}{\beta_{4}} \sqrt{\frac{\left(1-m^{2}\right)\left[6 \beta_{4}\left(\alpha_{1} \beta_{3}-\beta_{4} \gamma_{1}\right)^{2}+\left(6 \beta_{2} \beta_{4}+3 \beta_{3}^{2}\right) \lambda\right]}{10 \gamma_{2}\left(1+m^{2}\right)\left(\alpha_{1} \beta_{3}-\beta_{4} \gamma_{1}\right)}} \frac{e^{i \Theta} \operatorname{dn} \xi}{(1 \pm m \mathrm{sn} \xi)},
\end{aligned}
$$


Case 2.7: When $q_{0}=\frac{-\left(1-m^{2}\right)^{2}}{4}, q_{2}=\frac{m^{2}+1}{2}, q_{4}=\frac{-1}{4}, F(\xi)=(\operatorname{mcn} \xi \pm$ $d n \xi)$, we obtain

$E_{61,62}= \pm \frac{1}{2 \beta_{4}} \sqrt{\frac{2\left[6 \beta_{4}\left(\alpha_{1} \beta_{3}-\beta_{4} \gamma_{1}\right)^{2}-\left(6 \beta_{2} \beta_{4}+3 \beta_{3}^{2}\right) \lambda\right]}{5 \gamma_{2}\left(1+m^{2}\right)\left(\alpha_{1} \beta_{3}-\beta_{4} \gamma_{1}\right)}} e^{i \Theta}(m \operatorname{cn} \xi \pm \operatorname{dn} \xi)$,

$E_{63,64}= \pm \frac{1}{2 \beta_{4}} \sqrt{\frac{2\left[6 \beta_{4}\left(\alpha_{1} \beta_{3}-\beta_{4} \gamma_{1}\right)^{2}+\left(6 \beta_{2} \beta_{4}+3 \beta_{3}^{2}\right) \lambda\right]}{5 \gamma_{2}\left(1+m^{2}\right)\left(\alpha_{1} \beta_{3}-\beta_{4} \gamma_{1}\right)}} e^{i \Theta}(m \operatorname{cn} \xi \pm \operatorname{dn} \xi)$,

Case 2.8: When $q_{0}=m^{4}-2 m^{3}+m^{2}, q_{2}=6 m-m^{2}-1, q_{4}=\frac{-4}{m}$, then the solution of Eq. (2.4) is $F(\xi)=\frac{m \operatorname{cn} \xi \operatorname{dn} \xi}{\left(m \operatorname{sn}^{2} \xi+1\right)}$, and the solutions of Eq. (1.1) are $E_{65,66}(z, t)= \pm \frac{2}{\beta_{4}} \sqrt{\frac{m\left[6 \beta_{4}\left(\alpha_{1} \beta_{3}-\beta_{4} \gamma_{1}\right)^{2}-\left(6 \beta_{2} \beta_{4}+3 \beta_{3}^{2}\right) \lambda\right]}{5 \gamma_{2}\left(6 m-m^{2}-1\right)\left(\alpha_{1} \beta_{3}-\beta_{4} \gamma_{1}\right)}} \frac{e^{i \Theta} \operatorname{cn} \xi \operatorname{dn} \xi}{\left(1+m \operatorname{sn}^{2} \xi\right)}$,

$E_{67,68}(z, t)= \pm \frac{2}{\beta_{4}} \sqrt{\frac{m\left[6 \beta_{4}\left(\alpha_{1} \beta_{3}-\beta_{4} \gamma_{1}\right)^{2}+\left(6 \beta_{2} \beta_{4}+3 \beta_{3}^{2}\right) \lambda\right]}{5 \gamma_{2}\left(6 m-m^{2}-1\right)\left(\alpha_{1} \beta_{3}-\beta_{4} \gamma_{1}\right)}} \frac{e^{i \Theta} \operatorname{cn} \xi \operatorname{dn} \xi}{\left(1+m \mathrm{sn}^{2} \xi\right)}$,

where $\Omega$ and $a$ are given by Eq. (3.36) and Eq. (3.37) with the corresponding values of $q_{0}, q_{2}, q_{4}$ and $k, c$ are given by Eq. (3.32), Eq. (3.34). Other JEFs are omitted here for simplicity. As $m \rightarrow 1$, the JEFs Eq. (3.40) - Eq. (3.55) reduce to the following hyperbolic functions solutions:

$$
\begin{aligned}
& E_{69,70}(z, t)= \pm \frac{1}{\beta_{4}} \sqrt{\frac{\left[6 \beta_{4}\left(\alpha_{1} \beta_{3}-\beta_{4} \gamma_{1}\right)^{2}-\left(6 \beta_{2} \beta_{4}+3 \beta_{3}^{2}\right) \lambda\right]}{10 \gamma_{2}\left(\alpha_{1} \beta_{3}-\beta_{4} \gamma_{1}\right)}} e^{i \Theta} \tanh (\xi), \\
& E_{71,72}(z, t)= \pm \frac{1}{\beta_{4}} \sqrt{\frac{\left[6 \beta_{4}\left(\alpha_{1} \beta_{3}-\beta_{4} \gamma_{1}\right)^{2}+\left(6 \beta_{2} \beta_{4}+3 \beta_{3}^{2}\right) \lambda\right]}{10 \gamma_{2}\left(\alpha_{1} \beta_{3}-\beta_{4} \gamma_{1}\right)}} e^{i \Theta} \tanh (\xi), \\
& E_{73,74}(z, t)= \pm \frac{1}{\beta_{4}} \sqrt{\frac{\left[6 \beta_{4}\left(\alpha_{1} \beta_{3}-\beta_{4} \gamma_{1}\right)^{2}-\left(6 \beta_{2} \beta_{4}+3 \beta_{3}^{2}\right) \lambda\right]}{5 \gamma_{2}\left(\alpha_{1} \beta_{3}-\beta_{4} \gamma_{1}\right)}} e^{i \Theta} \operatorname{sech} \xi \\
& E_{75,76}(z, t)= \pm \frac{1}{\beta_{4}} \sqrt{\frac{\left[6 \beta_{4}\left(\alpha_{1} \beta_{3}-\beta_{4} \gamma_{1}\right)^{2}+\left(6 \beta_{2} \beta_{4}+3 \beta_{3}^{2}\right) \lambda\right]}{5 \gamma_{2}\left(\alpha_{1} \beta_{3}-\beta_{4} \gamma_{1}\right)}} e^{i \Theta} \operatorname{sech} \xi
\end{aligned}
$$


$E_{77,78}(z, t)= \pm \frac{1}{2 \beta_{4}} \sqrt{\frac{2\left[6 \beta_{4}\left(\alpha_{1} \beta_{3}-\beta_{4} \gamma_{1}\right)^{2}-\left(6 \beta_{2} \beta_{4}+3 \beta_{3}^{2}\right) \lambda\right]}{5 \gamma_{2}\left(\alpha_{1} \beta_{3}-\beta_{4} \gamma_{1}\right)}} \frac{e^{i \Theta} \tanh \xi}{(1 \pm \operatorname{sech} \xi)}$

$$
\begin{aligned}
& E_{79,80}(z, t)= \pm \frac{1}{2 \beta_{4}} \sqrt{\frac{2\left[6 \beta_{4}\left(\alpha_{1} \beta_{3}-\beta_{4} \gamma_{1}\right)^{2}+\left(6 \beta_{2} \beta_{4}+3 \beta_{3}^{2}\right) \lambda\right]}{5 \gamma_{2}\left(\alpha_{1} \beta_{3}-\beta_{4} \gamma_{1}\right)}} \frac{e^{i \Theta} \tanh \xi}{(1 \pm \operatorname{sech} \xi)}, \\
& E_{81,82}(z, t)= \pm \frac{2}{\beta_{4}} \sqrt{\frac{-\left[6 \beta_{4}\left(\alpha_{1} \beta_{3}-\beta_{4} \gamma_{1}\right)^{2}-\left(6 \beta_{2} \beta_{4}+3 \beta_{3}^{2}\right) \lambda\right]}{5 \gamma_{2}\left(\alpha_{1} \beta_{3}-\beta_{4} \gamma_{1}\right)}} \frac{e^{i \Theta} \operatorname{sech}^{2} \xi}{\left(\tanh ^{2} \xi+1\right)}, \\
& E_{83,84}(z, t)= \pm \frac{2}{\beta_{4}} \sqrt{\frac{-\left[6 \beta_{4}\left(\alpha_{1} \beta_{3}-\beta_{4} \gamma_{1}\right)^{2}+\left(6 \beta_{2} \beta_{4}+3 \beta_{3}^{2}\right) \lambda\right]}{5 \gamma_{2}\left(\alpha_{1} \beta_{3}-\beta_{4} \gamma_{1}\right)}} \frac{e^{i \Theta} \operatorname{sech}^{2} \xi}{\left(\tanh ^{2} \xi+1\right)},
\end{aligned}
$$

where the solutions in Eq. (3.56) and Eq. (3.57) are called dark soliton solutions and the solutions in Eq. (3.58) and Eq. (3.59) are called bright soliton solutions. Moreover, when $m \rightarrow 0$, we can obtain the triangular function solutions for Eq. (1.1) and we omitted these solutions here for simplicity.

\section{Conclusion}

In this paper, we used the F-expansion method with symbolic computations to solve a fourth order dispersion NLS equation with cubic-quintic nonlinearity, self-steeping and self-frequancy shift terms which describes the propagation of an optical pulse in optical fibers. According to this method, we investigated several types of exact solutions of Eq. (1.1). As results, exact solutions including JEF solutions, bright and dark solitary wave solutions are obtained. Although, the exact solutions of Eq. (1.1) with special parametric choices have studied by many researchers, we obtained in this research not only many exact solutions but also new types of exact solutions, to the best of our knowledge. These solutions may be useful for describing physical phenomena in nonlinear pulse propagation through optical fibers. Solutions in the limiting cases are studied and the properties of some solutions are also discussed. 

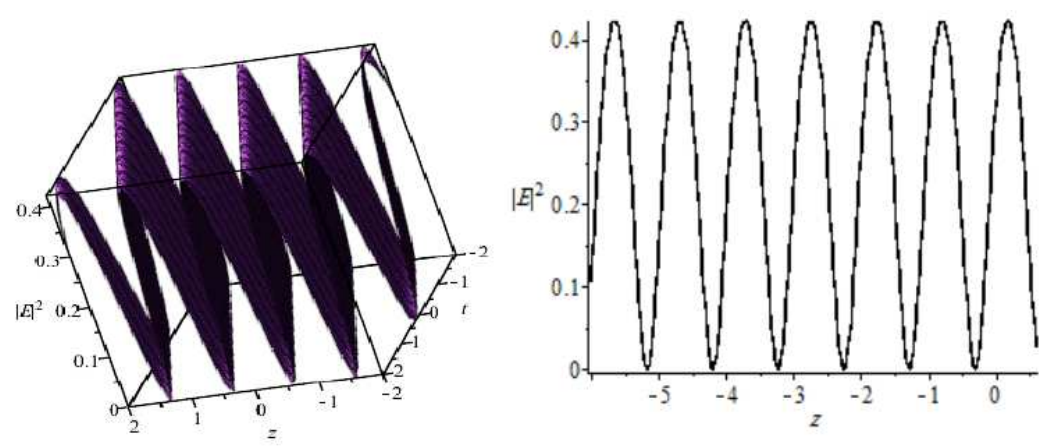

Figure 1(a): The intensity plot of the solution (3.12) and its position at $t=1$ with the parameters

$\beta_{2}=2, \beta_{3}=0.9, \beta_{4}=1.5, \gamma_{1}=1.5, \gamma_{2}=2, \alpha_{1}=-0.3, \alpha_{2}=-0.3, m=0.6$.
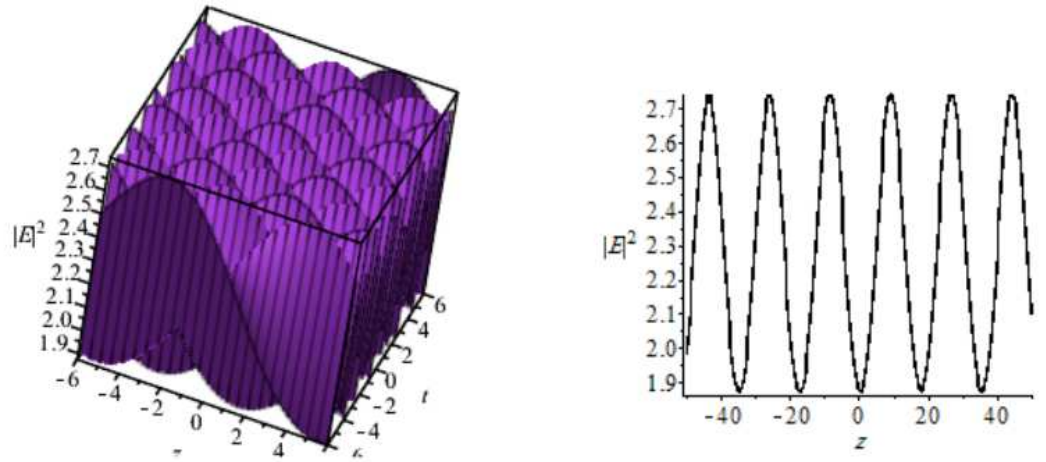

Figure 1(b): The intensity plot of the solution (3.13) and its position at $t=1$ with the same parameters as in Figure 1(a) but $\alpha_{1}=0.3, \alpha_{2}=0.3$.
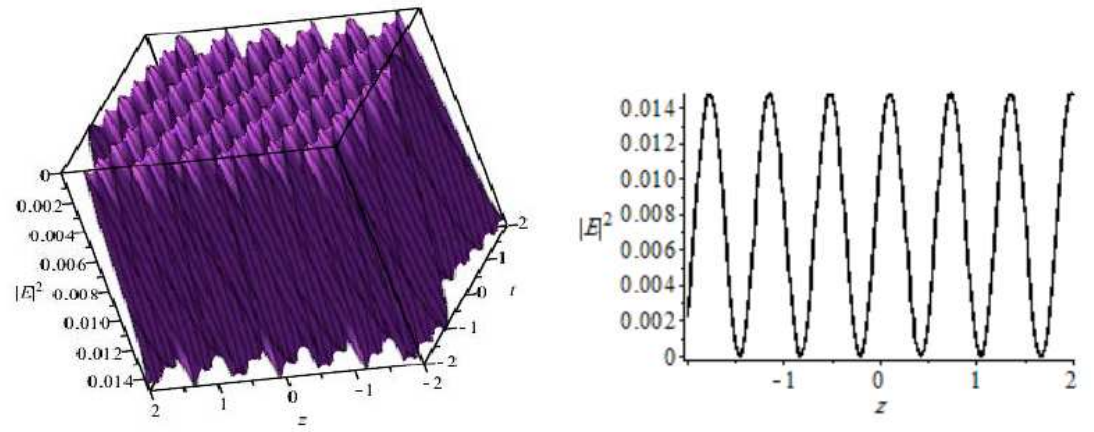

Figure 1(c): The intensity plot of the solution (3.15) and its position at $t=1$ with the same parameters as in Figure 1(a).

Figure 1 


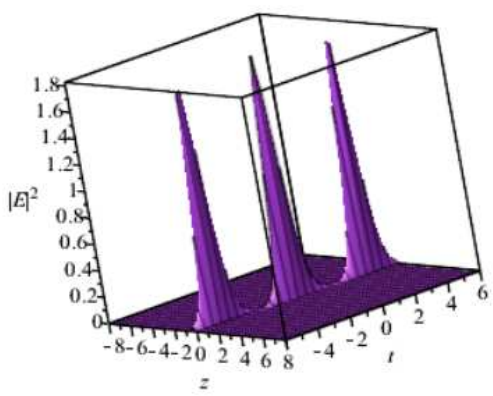

Figure 2(a): The intensity plot of the bright solitary wave solution (3.25) with the parameters $\beta_{2}=2, \beta_{3}=0.9, \beta_{4}=0.6$, $\gamma_{1}=1.5, \gamma_{2}=1.8, \alpha_{1}=0.3, \alpha_{2}=0.3$

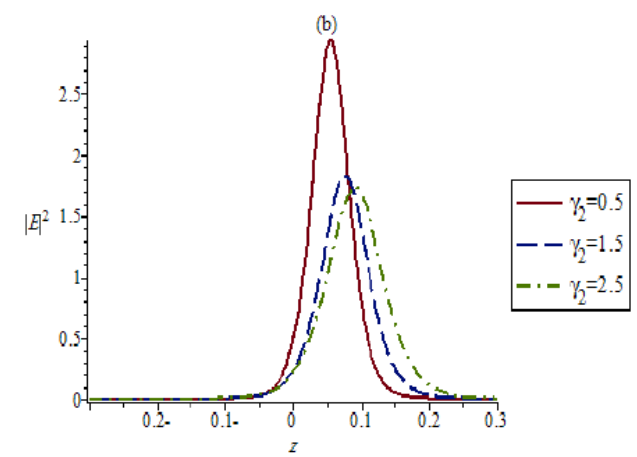

Figure 2(b): Its positions at $t=1$ for different values of $\gamma_{2}$.

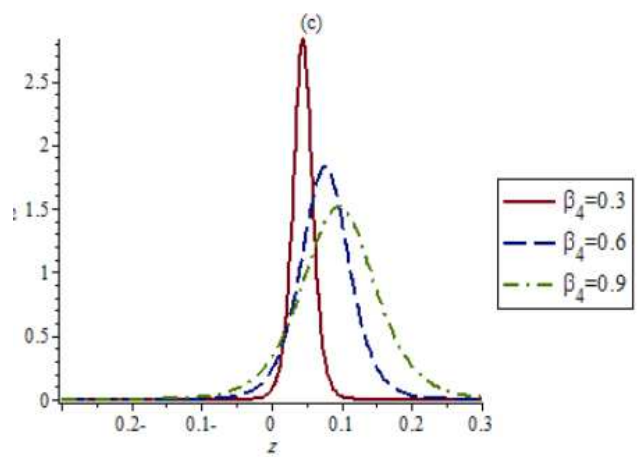

Figure 2(c): Its positions at $t=1$ for different values of $\beta_{4}$.

Figure 2 


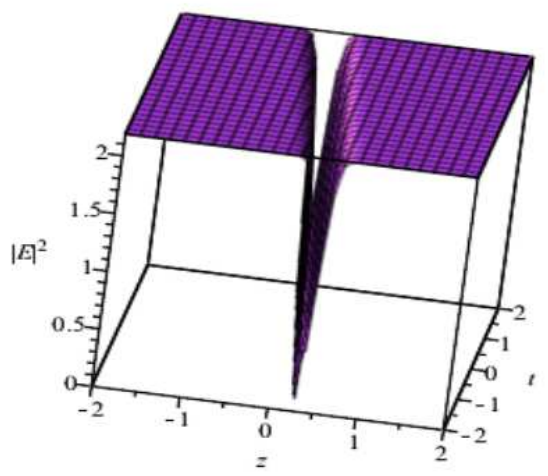

Figure 3(a): The intensity plot of the dark solitary wave solution (3.24) with the parameters $\beta_{2}=2, \beta_{3}=0.9, \beta_{4}=0.6$, $\gamma_{1}=1.5, \gamma_{2}=1.5, \alpha_{1}=-0.3, \alpha_{2}=-0.3$

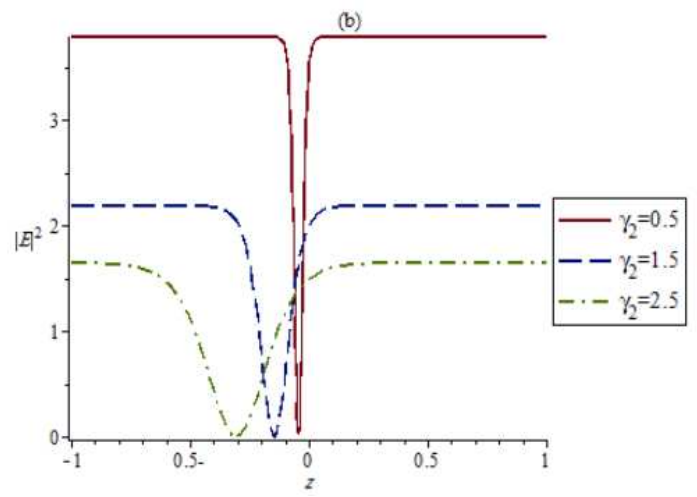

Figure 3(b): Its positions at $t=1$ for different values of $\gamma_{2}$.

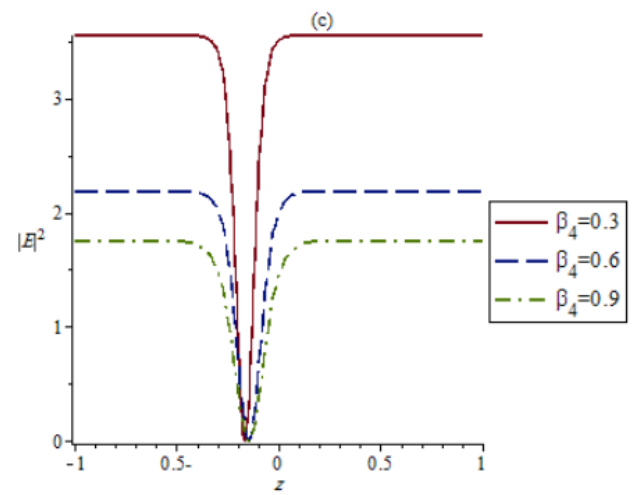

Figure 3(c): Its positions at $t=1$ for different values of $\beta_{4}$.

Figure 3 


\section{References}

[1] M. R. Miura, Bäcklund Transformation, Springer-Verlag, Berlin (1978).

[2] C. Rogers and W. F. Shadwick, Bäcklund Transformations, Academic Press, New York (1982).

[3] M. J. Ablowitz and P. A. Clarkson, Solitons, non-linear Evolution Equations and Inverse Scattering Transform, Cambridge Univ. Press, Cambridge (1991).

[4] W. Malfliet, Solitary wave solutions of nonlinear wave equations, American J. Phys. 60 (1992) 650-654.

[5] B. Q. Lu, Z.L. Pan, B.Z. Qu and X.F. Jiang, Solitary wave solutions for some systems of coupled nonlinear equation, Phys. Lett. A 180 (1993) 61-64.

[6] M. M. Hassan, Exact solitary wave solutions for a generalized KdV-Burgers equation Chaos, Solitons and Fractals 19 (2004) 1201-1206.

[7] S. Liu, Z. Fu, S.D. Liu and Q. Zhao, Jacobi elliptic function expansion method and periodic wave solutions of nonlinear wave equations, Phys. Lett. A 289 (2001) 69-74.

[8] Y. B. Zhou, M.L. Wang and Y. M. Wang, Periodic wave solutions to a coupled KdV equations with variable coefficients, Phys. Lett. A 308 (2003) 31-36.

[9] N. A. Kudryashov, On types of nonlinear nonintegrable differential equations with exact solutions, Phys. Lett. A 155 (1991) 269-275;

N. A. Kudryashov, One method for finding exact solutions of nonlinear differential equations, Comm. Non. Sci. Num. Simu 17 (2012) 2248-2253.

[10] M. M. Hassan, New exact solutions of two nonlinear physical models, Commun. Theor. Phys. 53 (2010) 596-604;

A. H. Khater, M. M. Hassan and D. K. Callebaut, Travelling wave solutions to some important equations of mathematical physics, Rep. Math. Phys. 66 (2010) 1 - 19.

[11] A. M. Wazwaz, The sine-cosine and the tanh method: reliable tools for analytic treatment of nonlinear dispersive equations, Phys. Lett. A 173 (2006) 150-164.

[12] E. G. Fan, Extended tanh- function method and its applications to nonlinear equations, Phys. Lett. A 277 (2000) 212-218.

[13] M. M. Hassan, M. A. Abdel-Razek and A. A.-H. Shoreh, New exact solutions of some $(2+1)$ - dimensional nonlinear evolution equations via extended Kudryashov method, Rep. Math. Phys. 74 (2014) 347-358.

[14] G. P. Agrawal, Nonlinear Fiber optics, Academic Press, New York (1995).

[15] A. Hasegawa and Y. Kodama, Solitons in Optical Communications, Oxford Univ. Press, New York, USA (1995).

[16] A. Ebaid and S. M. Khaled, New types of exact solutions for nonlinear Schrödinger equation with cubic nonlinearity, J. Comput. Appl. Math. 235 (2011) 1984-1992.

[17] J. Liang, H. Liu, F. Liu and L. Yi, Analytical solutions to the (3+1)-dimensional generalized nonlinear Schrödinger equation with varying parameters, J. Phys. A: Math. Theor. 42 (2009) 335204.

[18] C. Liu, Exact solutions for the higher-order nonlinear Schrödinger equation in optical fibres, Chaos, Solitons and Fractals 23 (2005) 949-955. 
[19] L.-P. Xu and J.-L. Zhang, Exact solutions to two higher-order nonlinear Schrödinger equations, Chaos, Solitons and Fractals 31 (2007) 937-942.

[20] J. Zhang and C. Dai, Bright and dark optical solitons in the nonlinear Schrödinger equation with fourth-order dispersion and cubic-quintic nonlinearity, Chin. Opt. Lett. 3 (2005) 295-298.

[21] Z. Li. L. Li, H. Tian and G. Zhou, New types of solitary wave solutions for the higher order nonlinear Schrödinger equation, Phys. Rev. Lett. 84 (2000) 4096-4099.

[22] H. Triki and T. R. Taha, The sub-ODE method and soliton solutions for higher order disperesive cubic-quintic nonlinear Schrödinger equation, Chaos, Solitons and Fractals 42 (2009) 1068-1072.

[23] Q. Zhou, D.Z. Yao and Z. Cui, Exact solutions of the cubic-quintic nonlinear optical transmission equation with higher-order dispersion terms and self-steeping term, J. Mod. Opt. 59 (2012) 57-60.

[24] W. P. Hong, Optical solitary wave solutions for the higher order nonlinear Schrödinger equation with cubic-quintic non-kerr terms, Opt. Commun. 194 (2001) 217-223.

[25] J.-L. Zhang, M.-L. Wang and X.Z. Li, The subsidary ordinary differental equations and the exact solutions of the higher order dispersive nonlinear Schrödinger equation, Phys. Lett. A 357 (2006)188-195.

[26] H. Triki and T. R. Taha, Solitary wave solutions for higher order nonlinear Schrödinger equation, Math. Computers in Simulation 82 (2012) 1333-1340.

[27] G. Q. Xu, New types of exact solutions for the fourth-order disperesive cubic-quintic nonlinear Schrödinger equation, Appl. Math. Computers 217 (2011) 5967-5971.

[28] H. Kumar and F. Ghand, Dark and bright solitary wave solutions of the higher order nonlinear Schrödinger equation with self-steeping and self-frequancy effects, J. Nonlinear Opt. Phys. and Materials 22 (2013) 1350001.

[29] M. Saha, A. K. Sarma. Solitary Wave solutions and modulation instability analysis of the nonlinear Schrödinger equation with higher order dispersion and nonlinear terms. Commun. Nonlinear Sci. Numer. Simulat. 18 (2013) 2420-2425.

[30] M. Abramowitz and I.A. Stegun, Handbook of Mathematical Physics, Dover, New York, (1965). 
